\title{
TRANS-PERINEAL ULTRASONOGRAPHIC MONITORING OF INTRAVAGINAL TRANSMISSIBLE VENEREAL TUMOUR REGRESSION WITH VINCRISTINE SULPHATE CHEMOTHERAPY IN DOGS: A STUDY OF TWELVE CASES
}

\author{
Bandula Rajapaksha, W. Chamara Ruwanmal Fernando and D.D.N. de Silva* \\ Veterinary Teaching Hospital, Department of Veterinary Clinical Sciences, \\ Faculty of Veterinary Medicine and Animal Science, \\ University of Peradeniya, Peradeniya, 20400, Sri Lanka.
}

\begin{abstract}
SUMMARY: Transmissible venereal tumour (TVT) grows mainly on the genitals of dogs. When such lesions develop in the vaginal passage, the process of clinical examination, diagnosis, and monitoring of therapeutic response are crucial in veterinary practice. The objective of this study was to investigate the usefulness of two-dimensional trans-perineal ultrasonography to monitor the therapeutic response of vincristine sulphate on intravaginal TVT in dogs. Twelve female dogs having lesions confirmed as TVT with cytological evaluation were used. TVT were categorized as multilobular $(n=7)$, solitary $(n=5)$, and heterogeneous $(n=12)$. The maximum transverse diameter of tumour was assessed ultrasonographically while treating with vincristine sulphate $0.025 \mathrm{mg} / \mathrm{kg}$, IV at weekly intervals. Owners were informed about the side effects of chronic vincristine sulphate chemotherapy such as development of pancytopenia, inappetence, and alopecia. Dogs were monitored for such adverse reactions during the study. The average maximum transverse diameter of tumour was $3.05 \pm 1.31 \mathrm{~cm}$ (range, 1.32 to $5.8 \mathrm{~cm}$ ) before the treatment. A variation of therapeutic effect on tumour size was observed with trans-perineal ultrasonography. A significant reduction of the lesions was recorded between two to five weeks of the treatment in 11 dogs $(P=0.00)$, with the highest reduction recorded one week after the treatment $(P=0.01)$. Eight dogs showed thickened and hyperechoic foci on the vaginal wall with the highest average thickness of $0.36 \pm 0.08 \mathrm{~cm}$ after the complete remission of TVT. Based on the results in this study, it could be concluded that trans-perineal ultrasonography is a simple and noninvasive diagnostic modality to characterize and monitor the therapeutic effect of vincristine sulphate in dogs affected with intravaginal TVT in clinical practice.
\end{abstract}

\section{INTRODUCTION}

Clinical examination, diagnosis, and monitoring of therapeutic response of deeply located intravaginal lesions of canine patients are crucial in veterinary practice. Transmissible venereal tumour (TVT) grows mainly on the genitals of dogs (Igor et al., 2012; Tella et al., 2004). TVT is usually transmitted from one dog to another during mating when abraded mucosa is exposed to the tumour of an infected dog (Otter et al., 2015). Further, Varela et al. (2013) suggested a possible acquisition of the tumour from the maternal genital TVT to puppies during the passage through the birth canal. In general, initial TVT lesions are superficial and 1-3 $\mathrm{mm}$ in diameter (Otter et al., 2015). Subsequently, multiple nodules fuse together forming larger, hemorrhagic, cauliflower-like, friable masses. The diameter of the masses could vary initially $(5-7 \mathrm{~cm})$ and progress gradually deeper into the mucosa as multilobular lesions with diameters exceeding 10-15 cm (Otter et al., 2015). When lesions develop in the vagina of a dog, several forms of manifestation including chronic vaginal bleeding, infertility, dystocia, and subsequent tumour metastasis could be encountered. The definitive diagnosis of TVT is based on history, physical examination, and cytological evaluation (Igor et al., 2012; Purohit et al., 2008; Thangathurai et al., 2008; Varela et al., 2013). However, when such lesions develop in anterior vagina, additional diagnostic aids are necessary to assess the severity and magnitude of the growth, and to plan and monitor the response to treatment.

Chemotherapy using vincristine sulphate is widely used as the therapeutic means for convenience of administration, efficacy, and relatively low cost (Purohit, 2008). Intravenous vincristine sulphate $0.025 \mathrm{mg} / \mathrm{kg}$, administered once a week for 3-6 weeks is reported to be one of the most effective chemotherapy protocols (de Silva, 1992; Otter et al., 2015; Purohit, 2008). It has been found to

Corresponding author: D.D.N. de Silva, e-mail: niranjalad@yahoo.com 
cause mitotic arrest and act on intercellular tubules (Tella et al., 2004). However, it is important to assess complete tumour remission accurately before treatment is terminated to prevent the risk of metastasis and the recurrence (Kose et al., 2013; Varela et al., 2013). Meanwhile, slight and transient normocytic normochromic anaemia and leucopenia have been observed in dogs due to the anti-mitotic effect of vincristine on bone marrow haemopoiesis (Tella et al., 2004). However, Tella et al., (2004) suggested that the side effects of vincristine sulphateare being tolerated by many dogs with the accurate dosage, short and well-spaced duration of the therapy.

Ultrasonography is considered as a user friendly, noninvasive, and real-time diagnostic modality in veterinary practice to diagnose, assess, and monitor the therapeutic effect on different tumours in dogs (Barthez et al., 1998; Feliciano et al., 2017; Naughton et al., 2012; Tagawa et al., 2016). The importance of the trans-perineal ultrasonographic characteristics of the birth canal of bitches during pregnancy and dystocia has been documented (Wickramasekara and de Silva, 2019). Furthermore, different ultrasonographic techniques and findings such as invasiveness, irregular contours, acoustic shadowing, and echo-texture of lesions are used in the differentiation of tumours (Feliciano et al., 2012; Lohet al., 2009; Pagani et al., 2016). However, a few studies have been carried out in relation to transperineal ultrasonographic characteristics of TVT in dogs. The objective of this study was to investigate the significance of using two-dimensional transperineal ultrasonography to characterize and monitor therapeutic effect on intravaginal TVT with vincristine sulphate chemotherapy in dogs.

\section{MATERIALS AND METHODS \\ Animals}

Twelve female dogs confirmed as having TVT by cytological evaluation were included in the present study. The dogs were German shepherd $(\mathrm{n}=2)$, Pomeranian $(\mathrm{n}=1)$, and mixed breed $(\mathrm{n}=9)$. The dogs were presented to the Veterinary Teaching hospital, University of Peradeniya with clinical signs namely, chronic intermittent reddish vulval discharge $(\mathrm{n}=11)$, urinary incontinence $(\mathrm{n}=3)$, stranguria $(\mathrm{n}=1)$, and infertility $(\mathrm{n}=1)$. The average age of dogs was $4.6 \pm 1.7$ years (range, 2 to 7 years) and the bodyweight $15.0 \pm 4.3 \mathrm{~kg}$ (range, 10 to $27.5 \mathrm{~kg}$ ). The patients were subjected to a thorough general clinical examination. As development of slight and transient pancytopenia have been reported with vincristine sulphate therapy (Tella et al., 2004), blood samples were collected from cephalic vein of each dog into EDTA coated tubes for complete blood count to monitor such changes. Written consent from owners was obtained before the use of their animals for the study. The study was carried out according to the ethical guidelines for animal studies at the Faculty of Veterinary Medicine and Animal Science, University of Peradeniya.

\section{Cytological evaluation}

Presence of TVT cells during cytological evaluation were used to identify dogs suffering from TVT in the present study. A cotton swab was inserted into the anterior vagina touching the masses suspected to be TVT. Three smears were prepared from collected sample from each dog and stained with Leishman stain for evaluation by light microscopy (x100 oil immersion). TVT was confirmed according to the cellular features described by Thangathurai et al. (2008). The cellular features looked for were the presence of oval to round nuclei, presence of coarsely aggregated chromatin, light blue cytoplasm with clear vacuoles, and numerous mitotic figures (Figure 1).

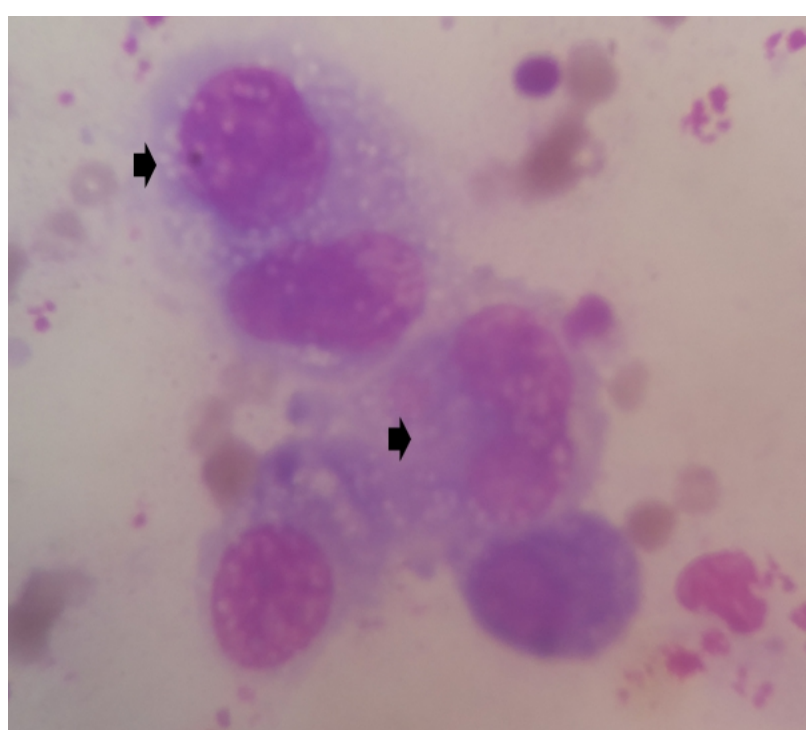

Figure 1. Round to polyhedral tumour cells (arrows) and polymorphonuclear nuclei containing coarsely aggregated chromatin at varying stage of mitosis in a smear prepared from a vaginal swab of a dog affected with TVT. The light blue cytoplasm of tumour cells contains typical vacuoles (Leishmann stain x 100).

\section{Ultrasonography procedure}

TVT cases which were confirmed with cytology evaluation were used for the ultrasonography examination in the present study. 
Two-dimensional trans-perineal sonograms were obtained using ultrasound scanner (MyLab30vet, Esaote, Genoa, Italy) with a phased-array transducer (Esaote PA 122, Esaote, Genoa, Italy) with frequency of $7.5 \mathrm{MHz}$. All the necessary dimensions were obtained from the sonographic images using electronic calipers. In each examined lesion, at least three measurements were taken in centimeters, and the means of all measurements were calculated as the final value. Real-time images were recorded on videotape and static images were sent to a computer equipped with specialized software for storage and off-line evaluation.

\section{Assessing the characteristics of TVT lesions with trans-perineal ultrasonography}

The transducer was positioned on the perivulva area with the dog in a lateral recumbency (Fimure ?)

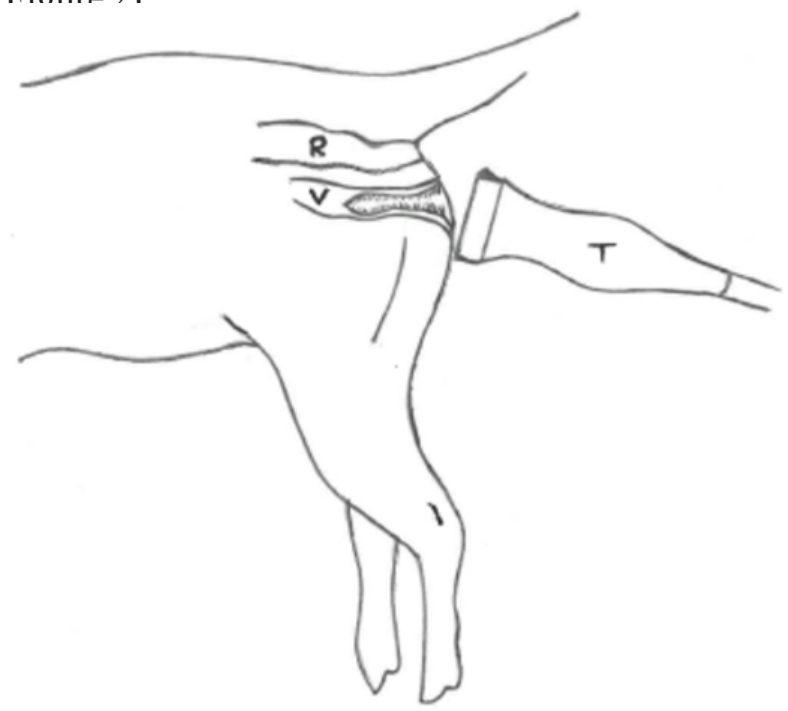

Figure 2. A schematic diagram shows the position of the transducer $(\mathrm{T})$ on the peri-vulva area with a dog in a lateral recumbency during trans-perineal ultrasonography. The transducer was positioned vertical in relation to the perineum of the dog and then slightly tilted dorsally to obtain the maximum visual length of the vaginal passage $(\mathrm{V})$.

The transducer was positioned vertical in relation to the perineum of the dog and then slightly tilted dorsally to obtain the maximum visual length of the vaginal passage. The maximum transverse diameter of tumour was measured from the wall of vagina to the edge of the tumour using electronic calipers. In addition, the following evaluation variables were recorded: lesion number (solitary, multiple), echotexture (heterogeneous, homogeneous) of lesions (Figure 3 ).
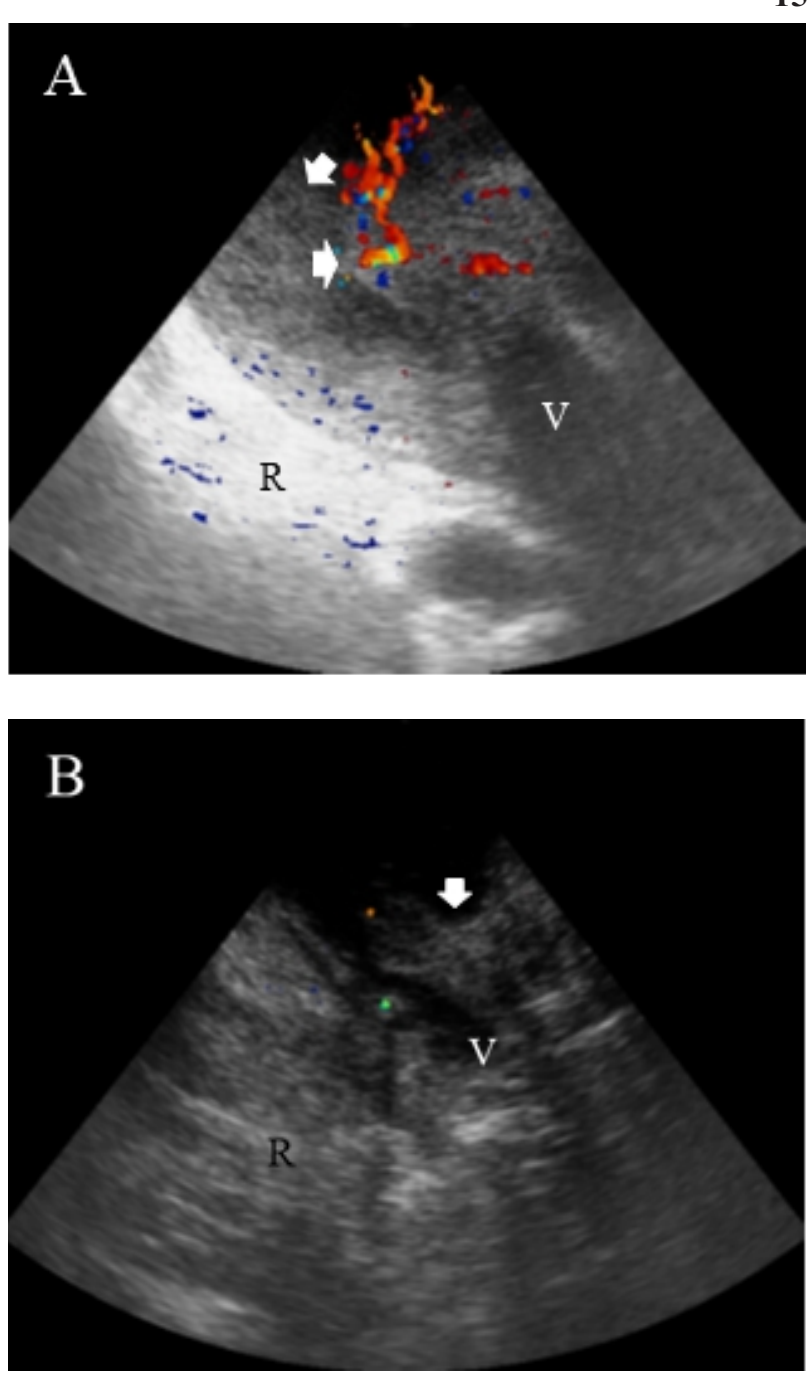

Figure 3. Representative trans-perineal ultrasonography images of TVT in (A) a 2-year old German shepherd showing multiple lesions (arrows) attached to the vaginal wall, and (B) a heterogeneous solitary lesion (arrow) with a broad base in a 1-year old mixed breed dog. Feacal matter in the rectum (R) can be seen dorsal to the vaginal passage. Dorsal is to the left, ventral is to the right, and cranial is to the bottom.

The color-flow Doppler ultrasonography was used subjectively to assess the vascularization in the lesion with a phased-array transducer (Esaote PA 122 , Esaote, Genoa, Italy) with frequency of 7.5 MHz. Trans-perineal ultrasonography was performed at intervals of one week until the TVT cells had disappeared at the vaginal cytological evaluation, and presence of any abnormality in the vaginal passage was recorded.

\section{Chemotherapy procedure}

As described by Tella et al. (2004), TVT cases were treated with vincristine sulphate (Belco 
Pharma PVT Ltd, India) $0.025 \mathrm{mg} / \mathrm{kg}$ that was slowly infused intravenously together with $0.9 \% \mathrm{NaCl}(10$ $\mathrm{ml} / \mathrm{kg}$ ). Before administering of vincristine sulphate, the dog owners were informed about the adverse effects of the chemotherapy such as, nausea, vomiting, weight loss, diarrhea, bloating, abdominal pain, hair loss, constipation, and loss of appetite (Tella et al., 2004). The most frequent complication of vincristine therapy is the occurrence of local tissue lesions caused by extravasation of the drug during intravenous application, resulting in the development of necrotic lesions with crusts in non-tumorous tissue (Otter et al., 2015). Therefore, precautions were taken to prevent extravascular leakage of vincristine sulphate when the chemotherapy was administered. Vincristine sulphate was administered at weekly
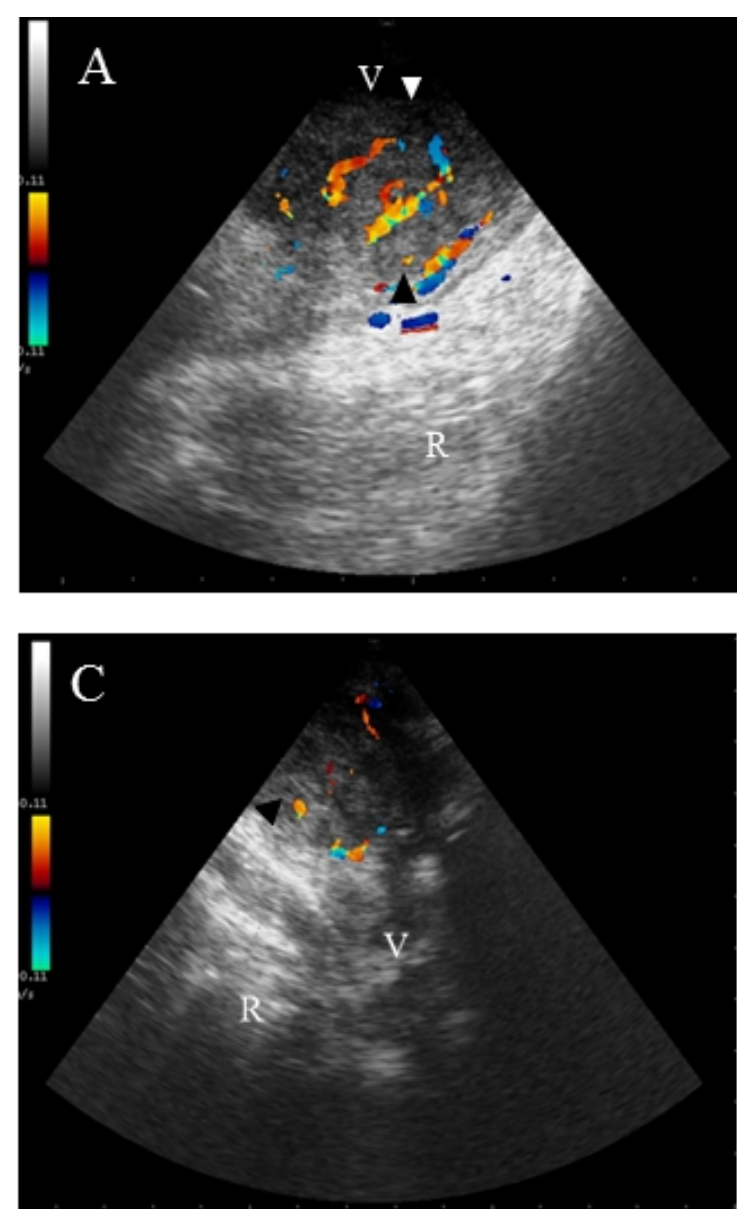

intervals until the tumour cells could not be detected with vaginal cytological evaluation.

\section{Statistical analysis}

All measurements were recorded on a Microsoft Excel spreadsheet. Statistical analysis was done using software Minitab ${ }^{\circledR}$ 18. Descriptive statistics, such as mean, range (minimum to maximum), and standard deviations of the mean were calculated for relevant parameters. The maximum transverse dimension of TVT lesion at first presentation was compared with the dimensions of the treated tumours. Therapeutic effects were categorized as complete regression, partial regression, or stable disease. Two sample paired t-test was done to compare the therapeutic effects. The $P$ value $<0.05$ was considered as statistically significant.
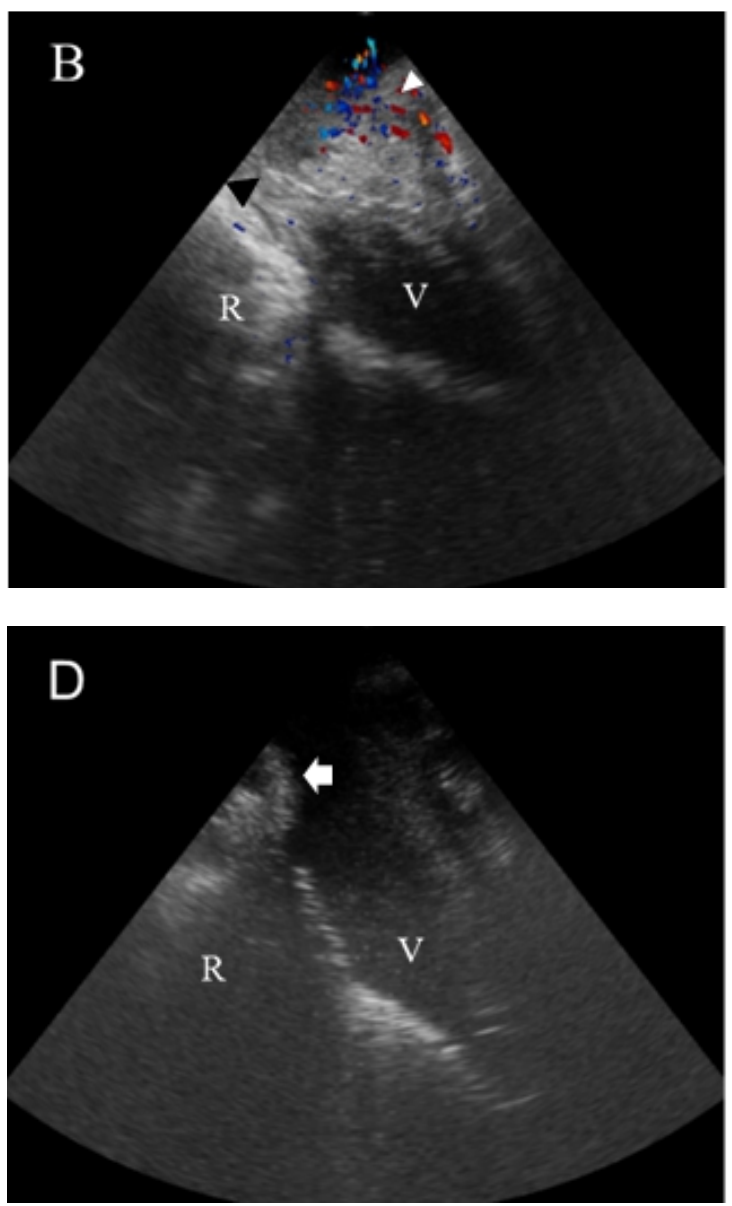

Figure 4. Trans-perineal ultrasonography images of TVT lesion of a 2-year mixed breed female dog. (A) A solitary, vascularized, heterogeneous mass lesion (arrow head) occluding the vaginal passage at the first day of presentation. (B) Partially regressed tumour one week after vincristine sulphate therapy. Vaginal passage (V) appears cranial to the tumour mass. (C) A significantly reduced tumour two weeks after the treatment, and (D) clear vaginal passage a week after the completion of chemotherapy protocol. The vaginal wall contains intermittent hyperechoic foci with irregular thickened areas (arrow). The maximum transverse diameter of the tumour was measured on the longest axis between the two arrow heads. Feacal matter in the rectum (R) can be seen dorsal to the vaginal passage. Dorsal is to the left, ventral is to the right, and cranial is to the bottom 


\section{RESULTS}

Trans-perineal ultrasonography was performed in 12 dogs who were suffering from TVT. Initially, the tumour appeared ultrasonographically as space occupying lesions in the vaginal passage. Tumours were broad based attached to the vaginal wall with clear vascularization in eight dogs. The average maximum transverse diameter of tumour was calculated as $3.05 \pm 1.31 \mathrm{~cm}$ (range, 1.32 to 5.8 $\mathrm{cm})$ just before the tumours were treated with vincristine sulphate. As shown in Figure 3, seven dogs had multilobular tumour masses in the vaginal passage, while 5 cases had a prominent solitary lesion (Figure 3A and 3B). All TVT lesions observed in the present study were heterogeneous in echotexture.

A variation in the reduction of tumour size was observed with trans-perineal ultrasonography in dogs affected with TVT after commencing treatment with vincristine sulphate. As shown in figure 4, a significant reduction of the lesions for vincristine sulphate was recorded between 2 to 5 weeks of treatment in $11 \operatorname{dogs}(P=0.00)$. The complete remission of tumour was observed in 2 dogs in 2 weeks after the commencement of the chemotherapy, while 6 dogs required 3 consecutive doses. Meanwhile, 4 doses of vincristine sulphate were needed for 2 dogs and 5 doses for 1 dog for the complete remission of TVT. Only one dog showed no considerable reduction of tumour after 3 consecutive doses of vincristine sulphate, thus it was considered as a partial regression. The average maximum size of tumour of second and third follow ups were measured as $1.80 \pm 0.88 \mathrm{~cm}$ (range, 0.5 to $3.8 \mathrm{~cm}$ ) and $1.12 \pm 0.92$ $\mathrm{cm}$ (range, 0.5 to $3.5 \mathrm{~cm}$ ) respectively. The highest reduction of maximum transverse diameter of tumour was recorded one week after the treatment $(P=0.01)$. Vascularization of the tumour progressively waned. Due to the shrinkage of the lesions, the vaginal passage could be clearly visualized ultrasonographically at the end of the chemotherapy protocol in 11 dogs (Figure 4D). As appeared in the Figure 4D, eight dogs showed thickened and hyperechoic foci on the vaginal wall at the end of the treatment, although cytological evaluation was negative for TVT cells. The average highest thickness of the vaginal wall of recovered dogs was $0.36 \pm 0.08 \mathrm{~cm}$.

In the present study, complete blood count of dogs were measured before each vincristine sulphate treatment. The hematological parameters did not show significant level of alteration to terminate the chemotherapyprotocol during the study period.

\section{DISCUSSION}

In the present study, the ultrasonographic characteristics of intravaginal TVT lesions in dogs were assessed using trans-perineal ultrasonography. Subsequently, the therapeutic effect on intravaginal TVT of vincristine sulphate was monitored by evaluating ultrasonographic characteristics and tumour size before, during weekly doses, and after completing the course of chemotherapy. It was revealed that most intravaginal TVT lesions of dogs were heterogeneous with irregular mucosal surface. Some conventional ultrasonographic findings may aid in establishing tumour type. Irregular echogenicity may occur because of necrosis, cyst formation, edema, hemorrhage, and calcification (Paulinelli et al., 2005; Tagawa et al., 2016). In addition, irregular margin, polymorphous shape, and heterogeneous internal characteristics were correlated with malignancy (Tagawa et al., 2016). The ultrasonographic characteristics of TVT recorded in the present study would be useful to assess the tissue composition of TVT and distinguish it from other perineal tumours. Furthermore, transperineal ultrasonography can be used effectively to identify degree of invasion of intravaginal lesion in relation to adjacent structures, especially the vagina, cervix, urethra, colon, and rectum. This would support to understand the clinical manifestation of the dogs affected with intravaginal masses. Observed clinical signs in dogs affected with intravaginal TVT in the present study such as urinary incontinence and stranguria probably may be due to the obstruction of urethral passage by the space occupying TVT masses, and damage to the blood vessels in it, respectively.

The trans-perineal ultrasonography can be used for evaluating therapeutic effect on intravaginal tumours. In the present study, the majority of dogs showed a significant reduction of tumour size with vincristine sulphate. The greatest reduction of maximum transverse diameter was observed one week after the commencement of chemotherapy. However, for the majority of dogs, continuation of treatment was required from one to five weeks until cytological evaluation became negative for TVT cells. Although the exact reason why some dogs required multiple doses of vincristine sulphate is not well known, factors such as the size of tumour, the degree of differentiation of tumor, and blood supply 
to the tumour tissue may affect the rate of remission of TVT. Trans-perineal ultrasonography could be used to reveal partial regression of TVT in a dog. Similar situations have been reported by Varela et al. (2013), which required adopting other chemotherapy protocols or surgical removal of tumours. Thus, one of the major advantages of trans-perineal ultrasonography is that it would enable early detection of ineffective chemotherapy protocols. This would minimize adverse effects of primary chemotherapy as non-responders could be easily identified. For example, it was observed that one dog in the present study showed no considerable reduction of tumour after 3 consecutive doses of vincristine sulphate. Therefore, the patient was prescribed with combination of treatment with vincristine sulphate, cyclophosphamide, and prednisolone.

In the present study, TVT lesions in 8 dogs showed the generalized vascularization throughout the lesions with colour Doppler ultrasound. The vascularization of tumour tissue reduced considerably with consecutive vincristine sulphate therapy. The colour Doppler ultrasonography allows quantification of vessel density which is also indicative of malignant lesions (Feliciano et al., 2012). Thus, the colour Doppler ultrasound can be considered as an additional diagnostic tool to monitor treatment response of TVT in association with transperineal ultrasonography. Meanwhile, trans-perineal ultrasonography revealed thickened, irregular, hyperechoic foci on the vaginal wall of dogs after the complete remission of TVT. As described by Otter et al. (2015), this could be due to the fact that tumour cells had been replaced by reactive fibrotic tissue and inflammatory cells which leads to vaginal wall hyperplasia (Figure 4D).

This study had several limitations. One of the major constraints of trans-perineal ultrasonography is limited view due to narrow window of pelvis of dogs (Figure 2). The phased-array transducer was used to investigate the far end of the vaginal passage as the beam from a transducer can be focused and swept electronically without moving the transducer (Thomas et al., 2013). Moreover, in the present study, TVT was selected to monitor therapeutic response as it is common among dogs and vincristine sulphate was considered as the first-line of treatment. However, further studies need to be carried out on different types of perineal tumours with different chemotherapeutic protocols to broaden the information of the present findings. In the present study, vaginal cytological evaluation was carried out to confirm TVT in dogs at weekly intervals until TVT cells disappeared with the chemotherapy. However, if cytological evaluation could be used in combination with histopathology, it would further increase the accuracy of detection in TVT.

In conclusion, this preliminary study has shown the potential diagnostic value of transperineal ultrasonography in dogs affected with intravaginal TVT. Furthermore, trans-perineal ultrasonography was used effectively in combination with cytological evaluation of tumour cells to monitor the therapeutic effect of vincristine sulphate on intravaginal TVT in dogs. On the basis of the information obtained in this study, trans-perineal ultrasonography can be used as a simple and noninvasive diagnostic modality to characterize and monitor the therapeutic effects of chemotherapy in dogs affected with intravaginal TVT in clinical practice.

\section{ACKNOWLEDGEMENTS}

The authors would like to thank clinical staff at the Veterinary Teaching Hospital, Department of Veterinary Clinical Sciences, University of Peradeniya, Sri Lanka, for their valuable assistance in carrying out this study. In addition, the authors would like to acknowledge the dog owners who allowed using their dogs in the present study.

\section{REFERENCES}

Barthez, P.Y., Nyland, T.G. and Feldman, E.C. (1998). Ultrasonography of the adrenal glands in the dog, cat, and ferret. Veterinary Clinics of North America: Small Animal Practice, 28, 869-885. https://doi.org/10.1016/S0195-5616(98)50082-4

De Silva, D.D.N. (1992). Vincristine sulphate for treatment of canine transmissible venereal tumour. Sri Lanka Veterinary Journal, 39, 7-13.

Feliciano, M.A.R., Uscategui, R.A.R., Maronezi, M.C., Simões, A.P.R., Silva, P., Gasser, B., Pavan, L., Carvalho, C.F., Canola, J.C. and Vicente, W.R.R. (2017). Ultrasonography methods for predicting malignancy in canine mammary tumors. PLOS ONE, 12, 1-14.

https://doi.org/10.1371/journal.pone.0178143 
Feliciano, M.A.R., Vicente, W.R.R. and Silva, M.A.M. (2012). Conventional and Doppler ultrasound for the differentiation of benign and malignant canine mammary tumours. Journal of Small Animal Practice, 53, 332-337.

https://doi-org.ezproxy.library.uq.edu.au/ 10.1111/j.1748-5827.2012.01227.x

Igor, U., Irena, C., Ksenija, I., Elena, A., Goran, N. and Plamen, T. (2012). Cytological diagnostic of canine transmissible venereal tumor - case report. Macedonian Veterinary Review, 35, 91-96.

Kose, A.M., Cizmeci, S.U., Aydin, I., Dinc, D.A., Maden, M. and Kanat, O. (2013). Disseminated metastatic transmissible venereal tumour in a bitch. Eurasian Journal of Veterinary Sciences, 29, 53-57. https://www.researchgate.net/publication/ 282246879

Loh, Z.H.K., Allan, G., Nicoll, R.G. and Hunt, G.B. (2009). Ultrasonographic characteristics of soft tissue tumours in dogs. Australian Veterinary Journal, 87, 323-329.

https://doi-

org.ezproxy.library.uq.edu.au/10.1111/j.17510813.2009.00460.x

Naughton, J.F., Widmer, W.R., Constable, P.D. and Knapp, D.W. (2012). Accuracy of three-dimensional and two-dimensional ultrasonography for measurement of tumor volume in dogs with transitional cell carcinoma of the urinary bladder. American Journal of Veterinary Research, 73, 19191924.

https://doi-org.ezproxy.library.uq.edu.au/10.2460/ ajvr.73.12.1919

Otter, W.D., Hack, M., Jacobs, J.J.L., Tan, J.F.V., Rozendaal, L. and Moorselaar, R.J.A.V. (2015). Effective treatment of transmissible venereal tumors in dogs with vincristine and IL2. Anticancer Research, 35, 3385-3392.

https://www.researchgate.net/publication/ 277405680

Pagani, E., Tursi, M., Lorenzi, C., Tarducci, A., Bruno, B.,Mondino, E.C.B. and Zanatta, R. (2016). Ultrasonographic features of adrenal glandlesions in dogs can aid in diagnosis. BMC Veterinary Research, 267, 1-9.

https://doi.org/10.1186/s12917-016-0895-1
Paulinelli, R.R., Freitas-Júnior, R., Moreira, M.A., Moraes, V.A., Bernardes-Júnior, J.R., Vidal Cda S., Ruiz, A.N. and Lucato, M.T. (2005). Risk of malignancy in solid breast nodules according to their sonographic features. Journal of Ultrasound in Medicine, 24, 635-641.

https://doi-org.ezproxy.library.uq.edu.au/10.7863/ jum.2005.24.5.635

Purohit, G.N. (2008). Canine transmissible venereal tumor: A review. Internet Journal of Veterinary Medicine, 6, 1-7.

https://www.researchgate.net/publication/ 337758242

Tagawa, M., Kanai, E., Shimbo, G., Kano, M. and Kayanuma, H. (2016). Ultrasonographic evaluation of depth-width ratio (D/W) of benign and malignant mammary tumors in dogs. Journal of Veterinary Medical Science, 78, 521-524.

https://doi.org/10.1292/jvms.15-0456

Tella, M.A., Ajala, O.O. and Taiwo, V.O. (2004). Complete regression of transmissible venereal tumour (tvt) in Nigerian mongrel dogs with vincristine sulphate chemotherapy. African Journal of Biomedical Research, 7, 133-138.

Thangathurai, R., Balasubramaniam, G.A.D.S., Balachandran, P.S.P. and Sivaseelan, S.M.B.M. (2008). Cytological diagnosis and its histological correlation in canine transmissible venereal tumour. Veterinarskiarhiv, 78, 369-376.

Thomas, L.S. and Peter, A.L. (2013). Ultrasound transducer selection in clinical imaging practice. Journal of Ultrasound in Medicine, 32, 573-582. https://doi-org.ezproxy.library.uq.edu.au/10.7863/ jum.2013.32.4.573

Varela, Y.D.M., de Queiroz, G.F., Filgueira, K.D., Reis, P.F.C.C. and Lima, R.K.R.(2013). Transmissible extragenital venereal tumor in impuberal canine. Brazilian Journal of Veterinary Pathology, 6, 123-127.

Wickramasekara, R.B.K. and De Silva, D.D.N. (2019). Trans-perineal ultrasonographic characteristics of the birth canal of bitches during pregnancy and dystocia. Japanese Journal of Veterinary Research, 67, 185-193. http://doi.org/10.14943/jjvr.67.2.185 